\title{
Electrical and Optical Properties of Carbon-Doped GaN Grown by MBE on MOCVD GaN Templates Using a $\mathbf{C C l}_{4}$ Dopant Source
}

Rob Armitage, Qing Yang, Henning Feick, Yeonjoon Park, and Eicke R. Weber

Presented at 2002 MRS Spring meeting, April 2-5, 2002, San Francisco, CA, USA

To be published in Defect- and Impurity-Engineered Semiconductors and Devices III, Proceedings of the 2002 MRS Spring meeting, San Francisco, CA, USA, 2-5 April, 2002 


\title{
Electrical and Optical Properties of Carbon-Doped GaN Grown by MBE on MOCVD GaN Templates Using a $\mathrm{CCl}_{4}$ Dopant Source
}

\author{
Rob Armitage, Qing Yang, Henning Feick*, Yeonjoon Park, and Eicke R. Weber \\ Department of Materials Science and Engineering, University of California, and \\ Materials Science Division, Lawrence Berkeley National Laboratory, Berkeley, CA \\ 94720
}

\begin{abstract}
Carbon-doped $\mathrm{GaN}$ was grown by plasma-assisted molecular-beam epitaxy using carbon tetrachloride vapor as the dopant source. For moderate doping mainly acceptors were formed, yielding semi-insulating GaN. However at higher concentrations p-type conductivity was not observed, and heavily doped films $\left(>5 \mu 10^{20} \mathrm{~cm}^{-3}\right)$ were actually $\mathrm{n}$ type rather than semi-insulating. Photoluminescence measurements showed two broad luminescence bands centered at 2.2 and $2.9 \mathrm{eV}$. The intensity of both bands increased with carbon content, but the $2.2 \mathrm{eV}$ band dominated in $\mathrm{n}$-type samples. Intense, narrow $(\sim 6 \mathrm{meV})$ donor-bound exciton peaks were observed in the semi-insulating samples.
\end{abstract}

\section{INTRODUCTION}

Carbon is a major residual impurity in gallium nitride grown by metal-organic chemical vapor deposition (MOCVD) [1], and has found practical use as an intentional dopant for semi-insulating base layers in transistor structures [2]. Despite this, the defects formed by carbon and their influence on $\mathrm{GaN}$ properties remain poorly understood.

Among possible defects, theorists considered $\mathrm{C}_{\mathrm{N}}, \mathrm{C}_{\mathrm{Ga}}$, carbon pairs, and $\mathrm{C}_{\mathrm{Ga}}$ in a DX-configuration $[3,4,5]$. However there is disagreement in calculated values for the defect formation energies and (in some cases) their levels in the $\mathrm{GaN}$ band gap. The problem is complicated since the energetically favored type of defect may depend on the growth conditions (Ga- or N-rich limit) and the Fermi level position.

Previous experiments on carbon-doped GaN yielded inconsistent results. Several groups pursued carbon as an alternative to magnesium for p-type doping, but only two succeeded [6,7]. One of these studies [7] involved zincblende-structure GaN and both used GaAs substrates. Other groups found that carbon compensated background donors to yield semi-insulating $\mathrm{GaN}[2,8,9]$, but could not obtain p-type conductivity. Still other studies found that carbon produced predominantly donors, or showed mixed acceptor/donor behavior depending on the dopant concentration [10,11]. A feature observed in all luminescence studies of heavily carbon-doped GaN was a deep-level yellow emission band [12].

Many earlier studies were complicated by factors such as the use of unconventional substrates, carbon dopant sources that introduced additional impurities, and relatively poor-quality $\mathrm{GaN}$ reference material. The present investigation avoids such

* On leave from center for advanced european studies and research (Caesar), Bonn, Germany 
complications. Device-quality MOCVD GaN templates are used for homoepitaxial overgrowth by molecular-beam epitaxy (MBE) and $\mathrm{CCl}_{4}$ vapor is used as a pure dopant source. The effects of the carbon concentration and silicon co-doping on GaN properties are investigated using photoluminescence and Hall effect/resistivity measurements.

\section{EXPERIMENTAL DETAILS}

The templates used for MBE growth were $2 \mathrm{~mm}$ thick semi-insulating GaN films grown by MOCVD on sapphire and purchased from Emcore. The backs of the substrates were coated with titanium thin films to facilitate heat absorption and temperature measurement inside the MBE chamber. Prior to loading, the substrates were cleaned in acetone and methanol, then fixed to the molybdenum sample holder with tantalum wires.

The MBE chamber is a modified Riber 1000 system equipped with effusion cells for gallium and silicon and a custom-built dc plasma source [13] for activated nitrogen. To dope the films with carbon, vapor from a vacuum vessel filled with liquid $\mathrm{CCl}_{4}$ (Sigma Aldrich, 99.9\%) was leaked through a needle valve into an injector tube pointed at the substrate. Flow rates were estimated from the chamber pressure and ranged from $0.01-1.0 \mathrm{sccm}$ as a function of the valve setting. The substrate temperature was estimated by a pyrometer, assuming an emissivity of 0.9 for the Ti film on the back of the substrate.

The MBE growth procedure involved outgassing the substrate in the nitrogen plasma beam for 5-10 min, followed by deposition of a nominally undoped layer for 5 min, followed by growth of the carbon-doped film. The temperature was $750 £$ for all steps. The growth rate was $250 \mathrm{~nm} /$ hour, limited by the active nitrogen flux. The thickness of the epilayers studied varied between 0.6 and $1.0 \mathrm{~mm}$. Gallium-rich conditions were maintained for all samples, which necessitated a range of $\mathrm{Ga}$ effusion cell temperatures between $995 \pm$ and $1025 \pm$ depending on the $\mathrm{CCl}_{4}$ flow rate, as discussed below. After growth, the samples were etched in a HF solution to remove the titanium from the substrate backside and any residual gallium droplets from the surface.

Photoluminescence was excited by a HeCd laser at $325 \mathrm{~nm}$ with an optical power of the order of $10 \mathrm{~mW}$. The light was diffracted by a $0.85 \mathrm{~m}$ double-grating monochromator and detected with a GaAs photo-multiplier using the standard lock-in technique. The temperature was controlled with a closed-cycle helium cryostat down to 14K. Room-temperature Hall effect measurements were performed on conducting samples using indium contacts and a magnetic field of $0.3 \mathrm{~T}$. Selected samples were sent to Applied Microanalysis Labs (Santa Clara, CA) for secondary ion mass spectroscopy (SIMS) to determine concentrations of carbon and residual impurities.

\section{RESULTS AND DISCUSSION}

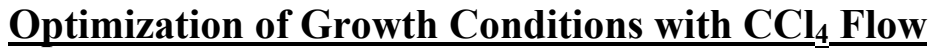

Abernathy et al. [6] reported that $\mathrm{CCl}_{4}$ doping drastically reduced the GaN growth rate due to parasitic etching, so our study first focused on this problem. Chlorine reacts with gallium to form highly volatile $\mathrm{GaCl}_{3}$, but the compound $\mathrm{GaN}$ is practically inert. Thus we hypothesized that the results of Abernathy et al. were caused by a competing reaction with chlorine which reduced the effective supply of gallium at the film surface, 
not actual etching of $\mathrm{GaN}$. This effect is undesirable not only due to the reduced growth rate, but also because the GaN quality suffers when grown in Ga-lean conditions.

Experiments with systematic variation of the gallium effusion cell temperature and $\mathrm{CCl}_{4}$ flow rate provided strong evidence for the above hypothesis. When the effusion cell temperature was kept constant and the $\mathrm{CCl}_{4}$ flow rate increased, the growth rate fell dramatically in agreement with the results of [6]. However, in experiments with fixed $\mathrm{CCl}_{4}$ flow, the growth rate increased with gallium cell temperature up to a maximum value corresponding to gallium-rich conditions. In this manner, carbon concentrations exceeding $5 \mu 10^{20} \mathrm{~cm}^{-3}$ were achieved with the same growth rate as for undoped samples.

A second concern with the $\mathrm{CCl}_{4}$ dopant source was possible incorporation of chlorine into the GaN films. Figure 1 shows a SIMS depth profile for a moderately carbon-doped semi-insulating sample. The carbon concentration is $1-2 \mu 10^{18} \mathrm{~cm}^{-3}$ while the chlorine concentration is negligible $\left(<3 \mu 10^{15} \mathrm{~cm}^{-3}\right)$. For very high $\mathrm{CCl}_{4}$ dopant flow rates the chlorine/carbon concentration ratio increases, but it is still less than 0.01 for the highest doping levels. The concentration of hydrogen also increases by a factor of 2-3 at very high doping levels, perhaps due to hydrocarbon impurities in the $\mathrm{CCl}_{4}$ source.

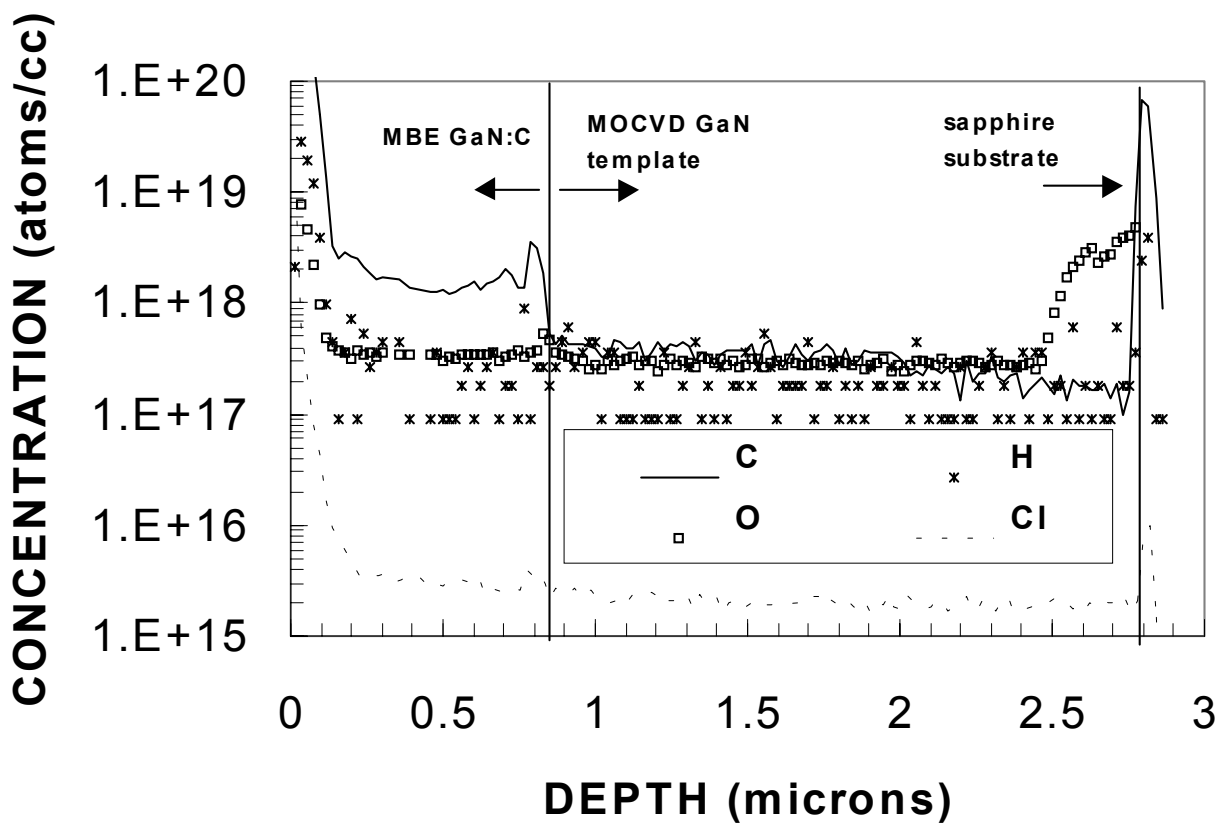

Figure 1. SIMS depth profile for a semi-insulating $\mathrm{GaN}$ film doped with $\mathrm{CCl}_{4}$.

\section{Electrical Properties}

Moderately carbon-doped samples $\left(1-2 \mu 10^{18} \mathrm{~cm}^{-3}\right)$ were semi-insulating with resistivity greater than $500 \mathrm{~W}-\mathrm{cm}$ (the measurement limit of the Hall-effect apparatus). This indicates that the concentration of $\mathrm{C}_{\mathrm{N}}$ acceptors exceeds the concentration of shallow donors (oxygen: $3-4 \mu 10^{17} \mathrm{~cm}^{-3}$, plus any shallow donors introduced by carbon). Even with $100 \%$ incorporation as $\mathrm{C}_{\mathrm{N}}$, a high resistivity would still be expected at this carbon concentration. Given the relatively high ionization energy of $\mathrm{C}_{\mathrm{N}}$, the free hole 
concentration would be just a few percent of the acceptor concentration. The resistivity of thin GaN layers with hole concentrations $<5 \mu 10^{16} \mathrm{~cm}^{-3}$ is too high to be measured in the Hall effect apparatus, given the low mobility $\left(<10 \mathrm{~cm}^{2} / \mathrm{V}-\mathrm{sec}\right)$ typical of $\mathrm{p}-\mathrm{GaN}$ and the high specific contact resistance.

More heavily carbon-doped samples $\left(2 \mu 10^{19} \mathrm{~cm}^{-3}\right)$ were also semi-insulating. Making a conservative estimate using the optical ionization energy of $230 \mathrm{meV}$, at least $1 \%$ of acceptors should be ionized at room temperature. Thus if carbon were incorporated predominantly as $\mathrm{C}_{\mathrm{N}}$, a hole concentration greater than $10^{17} \mathrm{~cm}^{-3}$ would be expected, giving measurable $\mathrm{p}$-type conductivity. Clearly a significant fraction of carbon must incorporate as other type(s) of defect rather than $\mathrm{C}_{\mathrm{N}}$. Birkle et al.[11] found a value of $\sim 50 \mathrm{meV}$ for the ionization energy of a carbon-related donor (perhaps $\mathrm{C}_{\mathrm{Ga}}$ ). If one uses this value for the donor and assumes $\mathrm{C}_{\mathrm{N}}$ is the only other possible carbon defect, a simple analysis shows that $51-65 \%$ of the total carbon must be incorporated in the form of $\mathrm{C}_{\mathrm{N}}$ to yield the observed semi-insulating behavior. Lower or higher fractions would result in measurable n- or p-type conductivity, respectively.

The most heavily carbon-doped sample $\left(>5 \mu 10^{20} \mathrm{~cm}^{-3}\right)$ actually showed n-type conductivity rather than semi-insulating behavior. A room temperature electron concentration of $1 \mu 10^{18} \mathrm{~cm}^{-3}$ and mobility of $25 \mathrm{~cm}^{2} / \mathrm{V}$-sec were observed. The low mobility is not surprising for a film expected to contain high concentrations of both donors and acceptors. The increased donor tendency is not due to a Fermi-level effect on defect formation energies, as the sample is n-type [3]. The higher donor fraction might result from kinetic limitations and/or exceeding the solubility limit on the nitrogen sublattice. The nature of defects responsible for the n-type conductivity remains unclear.

Co-doping experiments were carried out with $\mathrm{C}$ and $\mathrm{Si}$ in an attempt to reduce the $\mathrm{C}_{\mathrm{N}}$ ionization energy and achieve p-type GaN. According to the theory of Yamamoto and Katsuyama-Yoshida [14] the ideal ratio of donors to acceptors should be 1:2. This was not achieved in our experiments due the uncontrolled fraction of carbon incorporated as $\mathrm{C}_{\mathrm{N}}$. A sample with estimated Si doping of $3 \mu 10^{18} \mathrm{~cm}^{-3}$ and carbon concentration of 1$2 \mu 10^{19} \mathrm{~cm}^{-3}$ showed a free electron concentration of $1 \mu 10^{18} \mathrm{~cm}^{-3}$ and mobility of 66 $\mathrm{cm}^{2} / \mathrm{V}$-sec. The simple two-defect model suggests only half the total carbon was incorporated as $\mathrm{C}_{\mathrm{N}}$. Making no assumptions about alternative carbon defects, the $\mathrm{C}_{\mathrm{N}}$ concentration should be at least $2 \mu 10^{18} \mathrm{~cm}^{-3}$, i.e. $10-20 \%$ of the total carbon doping.

\section{Optical Properties}

Figure 2 a) shows low-temperature photoluminescence spectra for two semiinsulating films with carbon concentrations of $1-2 \mu 10^{18} \mathrm{~cm}^{-3}$ and $2 \mu 10^{19} \mathrm{~cm}^{-3}$. Both films have strong and narrow donor-bound exciton peaks, though they are degraded slightly in both width and intensity compared to an undoped sample. Two broad deep-level emission bands are evident with maxima at approximately 2.2 (yellow) and $2.9 \mathrm{eV}$ (blue). In the 


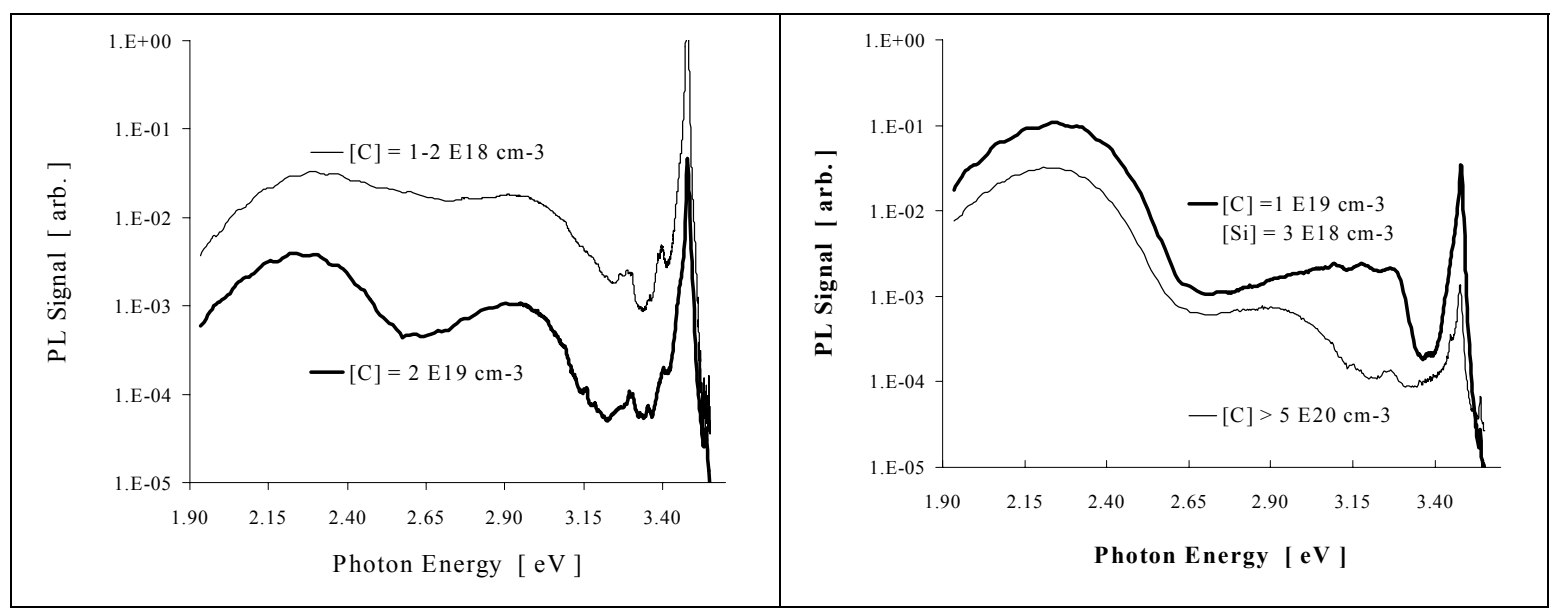

a) semi-insulating films

b) n-type films

Figure 2. Photoluminescence spectra taken at $14 \mathrm{~K}$ for carbon-doped GaN.

lightly doped sample the defect bands merge together, while they are better resolved in the moderately doped layer. The intensities of both defect bands relative to the donorbound exciton line clearly increase with carbon concentration. For the films in Fig. 2 a) the yellow luminescence (YL) is slightly stronger, though in other semi-insulating films with different $\mathrm{CCl}_{4}$ flow rates (not shown) the blue band is actually stronger.

Figure $2 \mathrm{~b}$ ) shows spectra for n-type samples, one very heavily carbon-doped $\left(>5 \mu 10^{20} \mathrm{~cm}^{-3}\right)$ and the other co-doped $\left(\sim 1-2 \mu 10^{19} \mathrm{~cm}^{-3} \mathrm{C}, \sim 3 \mu 10^{18} \mathrm{~cm}^{-3} \mathrm{Si}\right)$. The spectrum for the former sample is dominated by the YL, which exceeds the band-edge emission. The blue band is still present, but is of almost two orders of magnitude lower intensity than the YL. The silicon and carbon co-doped sample in Fig. 2 b) shows important differences relative to the n-type film doped only with carbon. In the co-doped sample the YL is dominant but strong band-edge emission is also observed. Also the blue defect band appears at higher energy and merges with donor-acceptor pair (DAP) peaks around $3.25 \mathrm{eV}$. Note that DAP transitions are evident only in the co-doped sample.

Comparing Figs. 2 a) and 2 b) it is evident that the relative YL intensity is strongest in n-type carbon doped samples. This result agrees with the widely-accepted mechanism for the YL proposed by van de Walle and Neugebauer [15] which involves a transition between a shallow donor and deep acceptor (Ga vacancy). However, semiinsulating carbon-doped films showed much stronger YL than the n-type reference sample despite the fact that the formation energy of $\mathrm{V}_{\mathrm{Ga}}$ is predicted to be high in semiinsulating GaN [15]. This apparent contradiction of the accepted YL mechanism might be explained if carbon doping introduces additional Ga vacancies above the equilibrium concentration for undoped GaN. Another possibility is that two YL mechanisms exist simultaneously, one involving $\mathrm{V}_{\mathrm{Ga}}$ and a second involving carbon defects. Additional work is needed to understand the deep-level luminescence in carbon-doped GaN.

\section{CONCLUSIONS}

Carbon-doped GaN films were grown homoepitaxially by plasma-assisted MBE on high-quality MOCVD GaN/sapphire templates using $\mathrm{CCl}_{4}$ as the dopant. Contrary to a previous report on the $\mathrm{CCl}_{4}$ source, the normal $\mathrm{GaN}$ growth rate was maintained at high 
doping levels provided the gallium flux was increased to compensate for gallium lost to a competing surface reaction. Chlorine contamination was negligible except at the highest $\mathrm{CCl}_{4}$ flow rates, and even then was less than $1 \%$ of the carbon concentration.

Carbon was found to form predominately acceptors for low doping levels, but the fraction incorporated on nitrogen sites decreased with increasing concentration, and donors were dominant in the most heavily doped films. The n-type conductivity of highly doped films cannot be explained by a Fermi-level dependence of the carbon defect formation energy.

In both n-type and semi-insulating carbon-doped films, two broad defect luminescence bands were observed centered around 2.2 and $2.9 \mathrm{eV}$ which increased in intensity with carbon concentration. The $2.2 \mathrm{eV}$ emission dominated the luminescence for n-type samples, while the $2.2 \mathrm{eV}$ and $2.9 \mathrm{eV}$ bands were of comparable intensity in semiinsulating samples. The fact that the semi-insulating samples showed strong YL suggests carbon either increases the concentration of gallium vacancies, or introduces another YL mechanism in addition to the generally accepted YL mechanism for undoped GaN.

\section{ACKNOWLEDGEMENTS}

This work was supported by the Director, Office of Science, Office of Basic Energy Sciences, Division of Materials Sciences and Engineering, of the U.S. Department of Energy under contract No. DE-AC03-76SF00098.

\section{REFERENCES}

1. S.J. Pearton, J.C. Zolper, R.J. Shul, and F. Ren, J. Appl. Phys. 86, 1 (1999)

2. H. Tang, J.B. Webb, J.A. Bardwell, S. Raymond, J. Salzman, and C. Uzan-Saguy, Appl. Phys. Lett. 78, 757 (2001)

3. P. Boguslawski and J. Bernholc, Phys. Rev. B 56, 9496 (1997)

4. J. Neugebauer and C. Van de Walle, in Proceedings of the $22^{\text {nd }}$ International Conference on the Physics of Semiconductors, p. 2327 (1995)

5. F. Fiorentini, F. Bernardini, A. Bosin, and D. Vanderbilt, in Proceedings of the $23^{\text {rd }}$ International Conference on the Physics of Semiconductors, p. 2877 (1997)

6. C.R. Abernathy, J.D. MacKenzie, S.J. Pearton, and W.S. Hobson, Appl. Phys. Lett. 66, 1969 (1995)

7. D.J. As and U. Kohler, J. Phys. Condens. Matter 13, 8923 (2001)

8. M. Sato, Appl. Phys. Lett 68, 935 (1996)

9. S. Shimizu and S. Sonoda, in Proc. $1^{\text {st }}$ Int. Workshop on Nitride Semiconductors, $\mathrm{p}$. 740 (2000)

10. R. Zhang and T.F. Kuech, Mat. Res. Soc. Symp. Proc. 482, 709 (1998)

11. U. Birkle, M. Fehrer, V. Kirchner, S. Einfeldt, D. Hommel, S. Strauf, P. Michler, and J. Gutowski, MRS Internet J. Nitride Semicond. Res. 4S1, G5.6 (1999)

12. T. Ogino and M. Aoki, Japan. J. Appl. Phys. 19, 2395 (1980)

13. A. Anders, N. Newman, M. Dickinson, E. Jones, P. Phatak, and A. Gassman, Rev. Sci. Instrum. 67, 905 (1996)

14. T. Yamamoto and H. Katayama-Yoshida, Jpn. J. Appl. Phys. 36, L180 (1997)

15. J. Neugebauer and C. Van de Walle, Appl. Phys. Lett. 69, 503 (1996) 